\title{
Simulation of the AGILE gamma-ray imaging detector performance: part I
}

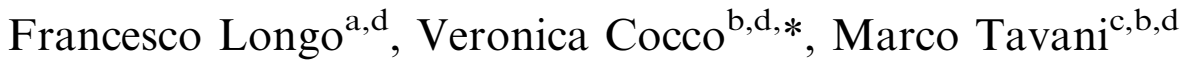 \\ ${ }^{a}$ Università degli Studi di Ferrara and INFN Sezione di Ferrara, Italy \\ ${ }^{\mathrm{b}}$ Università degli Studi "Tor Vergata" and INFN Sezione di Roma II, Italy \\ ${ }^{\mathrm{C}}$ Istituto di Fisica Cosmica, CNR, Milano, Italy \\ ${ }^{\mathrm{d}}$ Consorzio Interuniversitario Fisica Spaziale, Torino, Italy
}

Received 26 July 2001; received in revised form 25 October 2001; accepted 20 November 2001

\begin{abstract}
We present in this paper the results of a comprehensive GEANT simulation of the Gamma-Ray Imaging Detector (GRID) being developed for the AGILE space astrophysics mission. The GRID is designed to be sensitive in the $\sim 30 \mathrm{MeV}-50 \mathrm{GeV}$ energy range, with excellent imaging and timing capabilities, and with a very large field-of-view ( $\sim 3 \mathrm{sr}$ ). In this paper (Paper I) we present the GRID baseline geometry, a model for the charged particle and albedophoton backgrounds for an equatorial orbit of 550-600 km altitude, and the main results of the first level (Level-1 Trigger) on-board data processing. Our simulations show that the GRID Level-1 data processing is expected to be capable of decreasing by a factor of $\sim 20$ the charged particle background (from $\sim 2 \mathrm{kHz}$ to below $100 \mathrm{~Hz}$ ), and by a factor of $\sim 30$ the albedo-photon background. The gamma-ray photon detection efficiency by the imaging GRID is simulated to be particularly efficient, varying between $39 \%$ and $17 \%$ depending on photon energies and incident directions. (C) 2001 Elsevier Science B.V. All rights reserved.
\end{abstract}

PACS: 95.85.Pw; 95.55.ka; 95.75. $-\mathrm{z}$

Keywords: Gamma-ray instruments; Monte Carlo simulation

\section{Introduction}

AGILE is an Italian Space Agency (ASI) Small Scientific Mission dedicated to high-energy astrophysics [1,2]. The AGILE instrument is designed to detect and image photons in the $\sim 30 \mathrm{MeV}-$

\footnotetext{
*Corresponding author. Dip. di Fisica, Universita di Roma "Tor Vergata", Via della Ricerca Scientifica 1, 00133 Roma, Italy. Tel.: + 39-6-7259-4718; fax: + 39-6-7259-4647.

E-mail address: veronica.cocco@roma2.infn.it (V. Cocco).
}

$50 \mathrm{GeV}$ and $10-40 \mathrm{keV}$ energy bands, with excellent spatial resolution and timing capability, and a very large field of view covering $\sim \frac{1}{5}$ of the entire sky at energies above $30 \mathrm{MeV}$ [3]. Primary scientific goals include the study of AGNs, gamma-ray bursts, Galactic sources, unidentified gamma-ray sources, diffuse Galactic and extragalactic gamma-ray emission, high-precision timing studies and Quantum Gravity testing [3].

The AGILE Gamma-Ray Imaging Detector (GRID) consists of a Silicon-Tungsten Tracker, a 
Cesium Iodide Mini-Calorimeter (MC), an Anticoincidence system (AC) made of segmented plastic scintillators, and a Data Handling system (DH). The GRID is sensitive in the energy range $\sim 30 \mathrm{MeV}-50 \mathrm{GeV}$, and is designed to achieve an optimal angular resolution (source location accuracy $\sim 5-20^{\prime}$ for intense sources), a very large fieldof-view ( $\sim 3 \mathrm{sr}$ ), and a sensitivity comparable to that of EGRET for on-axis (and substantially better for off-axis) point sources. The optimal performance of the AGILE GRID requires an efficient on-board selection of events aimed at maximizing the background rejection and the transmission of cosmic photon events. This task is carried out by the Data Handling (DH) System.

In the current (Paper I) and companion [4] papers we present the main results concerning the simulation of GRID events induced by the particle/albedo-photon background and by the cosmic gamma-rays. We study in detail different levels of the GRID on-board trigger and data processing. For the Monte Carlo simulations we used the numerical code GEANT (version 3.21) [5] and related subroutines. Our numerical results are in very good agreement with CERN beam-test results obtained by our group with electrons and photons in the $0.1-1 \mathrm{GeV}$ energy range [6-8].

\section{Cosmic gamma-ray signal and expected particle and albedo-photon background}

\subsection{Cosmic gamma-ray flux}

The cosmic photon flux on the AGILE-GRID detector will be dominated by the diffuse gammaray emission, typically larger near the Galactic plane by a factor of 10 with respect to the highGalactic-latitude. This latter emission has a flux of $\Phi \simeq 10^{-5} \mathrm{ph} \mathrm{cm}^{-2} \mathrm{~s}^{-1} \mathrm{sr}^{-1}$ for photons above $100 \mathrm{MeV}$. Expected gamma-ray event rates for the AGILE-GRID depend on the pointed fields and can be given in the ranges (dominated by the diffuse gamma-ray emission): $0.02-0.1 \mathrm{ph} \mathrm{s}^{-1}$ for energies larger than $100 \mathrm{MeV}$ and $0.1-1 \mathrm{ph} \mathrm{s}^{-1}$ for energies between 10 and $100 \mathrm{MeV}$. The contribution of point-like gamma-ray sources adds to these rates without changing their order of magnitude.
In the simulations we considered 4 classes of cosmic gamma-ray photons, divided in low-energy photons $(25-50 \mathrm{MeV})$ and high-energy photons (400-1000 MeV); we used a power-law energy spectrum of index -2 , and run simulations for two extreme cases: an input geometry of incidence angle between 0 and $10^{\circ}$ off-axis, and an input geometry of incidence angle between 50 and $60^{\circ}$ off-axis.

\subsection{Charged particle background}

An equatorial orbit near $550 \mathrm{~km}$ of altitude for the AGILE mission will provide a relatively lowbackground environment. The charged particle background for this orbit is known to be relatively stable, with an increase by a factor 10-100 near the South Atlantic Anomaly (typically influencing about $10 \%$ of the orbital duration). Taking into account data from SAS-2 $[9,10]$ and BeppoSAX [11] missions, out of the South Atlantic Anomaly we expect an average rate of charged particle background above $\sim 1 \mathrm{MeV}$ of $\sim 0.3$ particles $\mathrm{cm}^{-2} \mathrm{~s}^{-1}$ (mostly electrons/positrons with a $\sim 10 \%$ contribution of protons).

In our simulations, based on spectral data from AMS [12-14] and MARYA [15], we considered the following background components: electrons (ELE), positrons (POS), primary high-energy protons (HE PROT) and secondary low-energy protons (LE PROT). We made the following assumptions: (1) we considered an "average equatorial position" and we assumed the AGILE $z$-axis pointed towards the sky for an unocculted GRID field of view; (2) we considered a spectrum averaged over the magnetic latitudes $\left|\lambda_{\text {mag }}\right|=0-11^{\circ}$ for secondary protons, and $\left|\lambda_{\mathrm{mag}}\right|=0-17^{\circ}$ for electrons and positrons (values compatible with the AGILE equatorial orbit), regardless of longitude dependence; (3) we considered an isotropic distribution on a virtual sphere containing the satellite for particles below the geomagnetic cutoff (albedo leptons and secondary protons), and an isotropic distribution emitted from the upper hemisphere for the primary protons above the cutoff. The adopted particle spectra, which are fits of the AMS and MARYA data (calculated in [16]) are shown in Fig. 1. Their fluxes are normalized to 


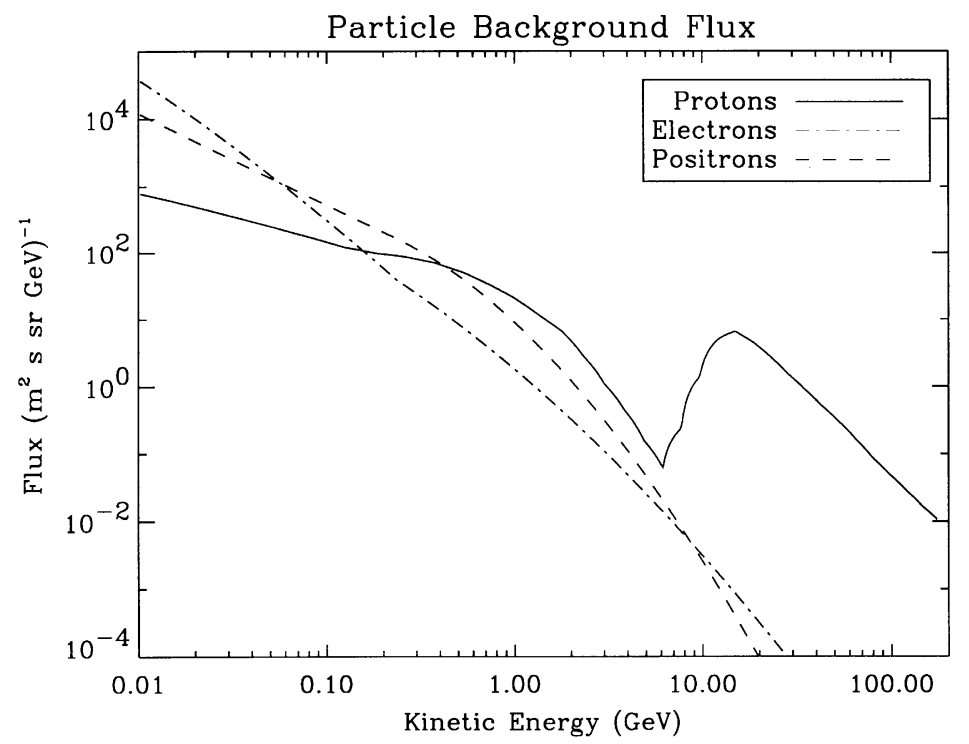

Fig. 1. Charged particle background for an equatorial orbit of $550 \mathrm{~km}$ (results from Ref. [16]) that we assumed in our calculations. Electron, positron and proton spectra were obtained by combined AMS-Shuttle Flight data and MARYA data. All particle components have an approximately isotropic distribution of incidence angles on the GRID, except the primary high-energy protons above the geomagnetic cutoff of $\sim 6 \mathrm{GeV}$.

Table 1

Integrated particle flux rates

\begin{tabular}{lcccc}
\hline Particles & $E_{\min }$ & $\begin{array}{l}E_{\max } \\
(\mathrm{GeV})\end{array}$ & $\mathscr{F}\left(\mathrm{cm}^{-2} \mathrm{~s}^{-1} \mathrm{sr}^{-1}\right)$ & $\pi \mathscr{F}\left(\mathrm{cm}^{-2} \mathrm{~s}^{-1}\right)$ \\
\hline Electrons & $10 \mathrm{MeV}$ & 10 & $3.76 \times 10^{-2}$ & 0.1180 \\
Positrons & $10 \mathrm{MeV}$ & 10 & $2.67 \times 10^{-2}$ & 0.0840 \\
Albedo protons & $10 \mathrm{MeV}$ & 6 & $9.04 \times 10^{-3}$ & 0.0284 \\
Primary protons & $6 \mathrm{GeV}$ & 180 & $9.99 \times 10^{-3}$ & 0.0314 \\
\hline
\end{tabular}

the values indicated in Table 1 and the total incidence rate on a surface is assumed to be $\sim 0.3$ particles $\mathrm{cm}^{-2} \mathrm{~s}^{-1}$ for particle kinetic energies above $\sim 10 \mathrm{MeV}$.

\subsection{Albedo gamma-ray background}

The interaction of the charged cosmic-rays with the upper atmosphere induces a relatively strong and non-isotropic gamma-ray background. It peaks near the Earth horizon (corresponding to a zenith angle $\theta=112^{\circ}$ for an orbit of $550 \mathrm{~km}$ altitude), and has a characteristic East-West asymmetry (by a factor of $\sim 4$ in intensity near $40 \mathrm{MeV}$ ) [17].

In our simulations, we used a simplified but representative model of the albedo-photon emission, and we considered two geometries: (1) albedo-photons reaching the GRID "from below", a situation valid for the unocculted portions of the AGILE orbit (ALB-1 PHOT); (2) Earth covering half of the AGILE FOV, with a large fraction of albedo-photons reaching the GRID "from one lateral side" (ALB-2 PHOT). Based on balloon flight data [18-22] and SAS-2 data [17], we have assumed the following simplified spectrum 
representing the average of the emission over the whole solid angle subtended by the Earth surface ( $\sim 4 \mathrm{sr})$ at the height of $550 \mathrm{~km}$ :

$\frac{\mathrm{d} N}{\mathrm{~d} S \mathrm{~d} t \mathrm{~d} E \mathrm{~d} \Omega}=\left\{\begin{array}{l}0.08 E^{-1.4} \mathrm{~cm}^{-2} \mathrm{~s}^{-1} \mathrm{sr}^{-1} \mathrm{MeV}^{-1} \\ 0.5 E^{-2.2} \mathrm{~cm}^{-2} \mathrm{~s}^{-1} \mathrm{sr}^{-1} \mathrm{MeV}^{-1}\end{array}\right.$

for $1 \mathrm{MeV}<E<10 \mathrm{MeV}$

for $10 \mathrm{MeV}<E<100 \mathrm{GeV}$ with a total flux $\mathscr{F}=0.15 \mathrm{~cm}^{-2} \mathrm{~s}^{-1} \mathrm{sr}^{-1}$ in the energy range $1 \mathrm{MeV}-100 \mathrm{GeV}$.

\subsection{Background rejection}

The ratio of charged particle events (penetrating the AC) to cosmic gamma-ray photon events is typically of order $10^{3}-10^{4}$ for photons of energy 20 $100 \mathrm{MeV}$, and $10^{4}-10^{5}$ for photons above $100 \mathrm{MeV}$. Albedo-photons are larger than cosmic gamma-ray photons by factors between 10 and 100 (depending on the pointing geometry and energy range).

Clearly, is necessary to 'filter' the GRID events. This task is carried out by a hardware-implemented fast logic ("Level-1 trigger"), and by a set of asynchronous software algorithms and CPU processing ("Level-2 processing"). The adopted requirements for the DH processing depend on the downlink telemetry rate, assumed to be $512 \mathrm{kbit} / \mathrm{s}$ during the $10 \mathrm{~min}$ duration of satellite visibility from the ASI ground station in Malindi (Kenya). A main aim of the AGILE DH system is to provide an on-board filtering of events reducing the background rate to an acceptable value within a factor of 10-100 of the cosmic gamma-ray photon rate. A final filtering of the particle and albedo-photon background will be carried out on the ground by a dedicated software. The charged particle and the albedo-photon background models described in the previous sections were used in Monte Carlo simulations in order to optimize the DH processing.

\section{The AGILE model and GRID detection assumptions}

\subsection{The AGILE model}

A correct mathematical and physical model of the AGILE payload and spacecraft with all their relevant components is crucial to evaluate the GRID behaviour and performance. A general description of the AGILE instrument can be

found in $[1,3]$. Simulation programs consider the following AGILE components (see Fig. 2): the spacecraft (bus) MITA and the AGILE payload, consisting of the CsI Mini-Calorimeter, the Silicon-Tungsten Tracker, the Anticoincidence system, the X-ray detector (Super-AGILE), the thermal shield, the mechanical structure and the lateral electronics boards. The AGILE components were modeled as follows:

(1) MITA spacecraft: The spacecraft (bus) MITA is represented by a box, made of carbonfibers, containing 10 equidistant aluminum layers inserted in thin $(100 \mu \mathrm{m})$ carbon-fiber structures; every aluminum layer is $1.1 \mathrm{~cm}$ thick for a total weight of $128 \mathrm{~kg}$.

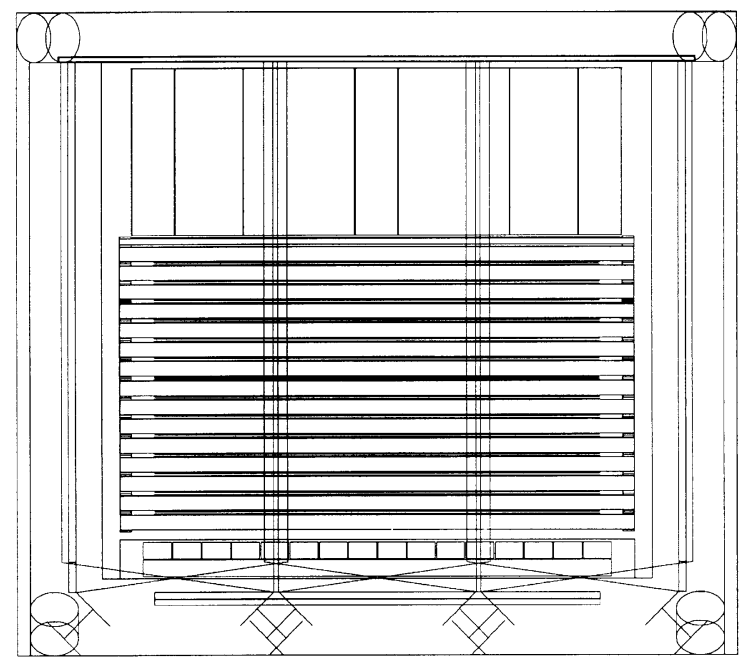

Fig. 2. AGILE payload: from the bottom the different volumes are: the DH box, the 2 planes of the Mini-Calorimeter, the 14 layers of the Silicon Tracker, Super-AGILE, the top AC panel. Also the lateral AC panels with the photomultipliers and the thermal shield are visible. The overall dimensions are: length of $62.55 \mathrm{~cm}$, width of $62.55 \mathrm{~cm}$ and height of $54.02 \mathrm{~cm}$. The GRID active volume dimensions are: $38.06 \times 38.06 \times 21.078 \mathrm{~cm}^{3}$ including the Tracker planes from the top sheet of the first tungsten layer to the bottom sheet of the last silicon- $y$ plane. 
(2) Mini-Calorimeter: The Mini-Calorimeter is modeled by two planes, each containing $16 \mathrm{CsI}$ bars, oriented as the $y$-axis in the upper plane and as the $x$-axis in the lower plane. The CsI bars are $1.4 \mathrm{~cm}$ thick and have a pitch of $2.4 \mathrm{~cm}$. They are inserted in a carbon-fiber structure $0.1 \mathrm{~cm}$ apart from each other. The distance between the $X$ layer and the $Y$ layer is assumed to be $0.1 \mathrm{~cm}$. The MiniCalorimeter is placed directly above the bus MITA, at a $0.5 \mathrm{~cm}$ distance from the Silicon Tracker.

(3) Silicon Tracker: The Silicon Tracker is made of 14 detection planes, with a distance between consecutive planes of $1.6 \mathrm{~cm}$ and a distance between the $X$ and $Y$ layers in each plane of $0.16 \mathrm{~cm}$; the first 12 planes contain also a tungsten layer each $(245 \mu \mathrm{m}$ thick $)$. Each silicon layer is composed of 16 tiles of $9.5 \times 9.5 \mathrm{~cm}^{2}$ each, with a pitch of $121 \mu \mathrm{m}$ (each tile contains 768 strips) and a thickness of $410 \mu \mathrm{m}$. In our model there are also the honeycomb support, the aluminum inserts connecting the tracker planes with the main structure of the satellite, and the Front-End electronics chips (3 TA1 for each ladder). Every tracker plane is composed by a Carbon-fiber structure $(500 \mu \mathrm{m}$ thick) supporting an aluminum honeycomb. Under the carbon-fiber are glued the tungsten layer (for the first 12 planes), one layer of kapton $(100 \mu \mathrm{m}$ thick) with a copper coating (15 $\mu \mathrm{m}$ thick) and the $X$ silicon layer. Under the $X$ layer there are the $Y$ layer and another kaptoncopper layer, which in the reality are glued to the next plane structure. On the same structure are glued also the ceramic hybrids which sustain the TA1 silicon chips. The global structure is composed by $14 X$ and $Y$ detectors layers and by 15 structure supports; the total tracker height is $24 \mathrm{~cm}$.

(4) Anticoincidence system: The AC system is made of a top panel of plastic scintillator $\left(54 \times 54 \times 0.5 \mathrm{~cm}^{3}\right)$ and 3 overlapping panels for each side of the AGILE Tracker $(0.6 \mathrm{~cm}$ thick, $18.1 \mathrm{~cm}$ large and $44.4 \mathrm{~cm}$ high). The AC plastic panels are inserted in a carbon-fiber structure $(500 \mu \mathrm{m}$ thick) and are supported by some aluminum inserts; the trapezoidal form of the lateral panels is described in a proper way. We have inserted also a schematic description of the photomultipliers and their casings. The AC system surrounds the Mini-Calorimeter, the Silicon Tracker and Super-AGILE, and is positioned above the bus MITA. Fig. 3 shows the adopted model.

(5) X-ray detector (Super-AGILE): The X-ray detector is made substantially by these elements: a silicon detection layer, a collimation system, a gold mask. A tungsten ring is positioned below the $\mathrm{Si}$ detection plane in order to reduce the diffuse photon background. The detection layer is divided in 16 tiles with the same characteristics of the tracker tiles, described in a realistic way, with the silicon microstrips and the Front-End electronics. We have modeled also the honeycomb and carbon-fiber support structure; the detectors and the support of the coded mask are glued on this structure. The silicon detector is placed over the tracker, while the distance between the silicon detector and the gold mask ( $90 \mu \mathrm{m}$ thick) is fixed to be $14 \mathrm{~cm}$. Also the collimation system, made of an ultra-light carbon-fiber structure $(500 \mu \mathrm{m}$ thick), is described in great detail (Fig. 4). Its internal panels are coated with a $75 \mu \mathrm{m}$ thick gold layer, in order to reduce the contribution from the

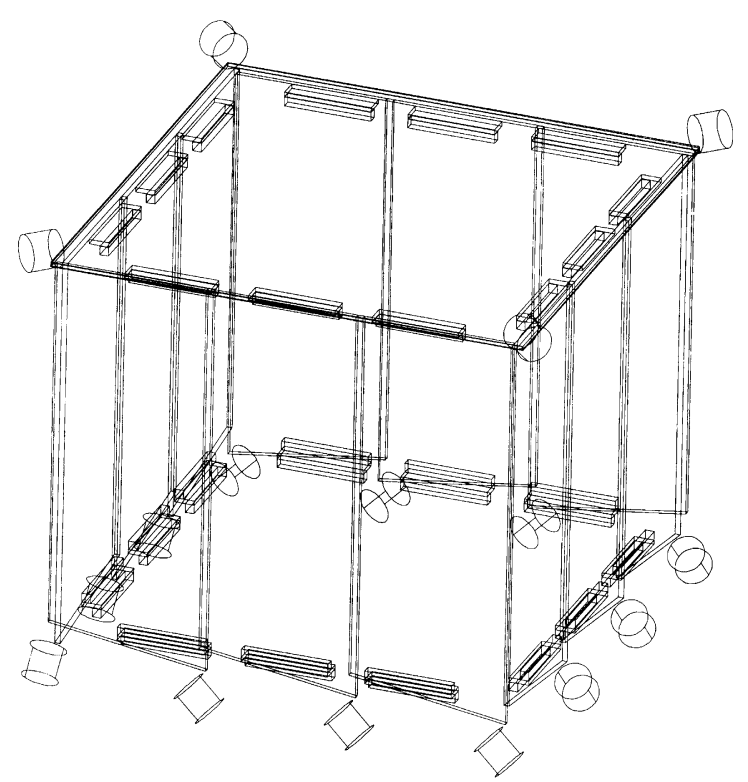

Fig. 3. Anticoincidence system. The overall dimensions are: length of $62.25 \mathrm{~cm}$; width of $62.25 \mathrm{~cm}$; height of $53.87 \mathrm{~cm}$. 


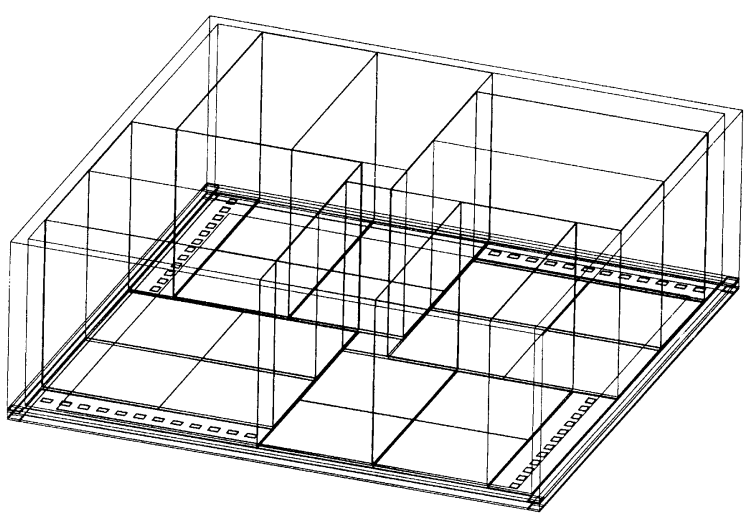

Fig. 4. Super-AGILE. Overall dimensions: length of $44 \mathrm{~cm}$; width of $44 \mathrm{~cm}$; height of $14.77 \mathrm{~cm}$.

diffuse cosmic X-ray background. The distance between the gold mask and the top AC panel is $0.5 \mathrm{~cm}$.

(6) Thermal shield: The thermal shield is modeled as an external Teflon layer (1 mm thick) plus an internal aluminum layer $(10 \mu \mathrm{m}$ thick $)$. It covers the Mini-Calorimeter, the Tracker, SuperAGILE and the AC system.

(7) Mechanical structure: The description of the payload is completed by a preliminary model for the general support structure: four aluminum legs connected by other aluminum bars.

There is also a preliminary description of the Front-End Electronics (FEE) and of the trigger vertical boards, one for each lateral side of the payload, and one for the DH box, under the MiniCalorimeter. They are described as some layers of silicon, ceramics and copper.

\subsection{Silicon Tracker capacitive coupling, floating strip readout, and Gaussian noise}

The electronic system for the AGILE Silicon Tracker is based on the "floating strip" readout. This implies that only one out of two contiguous Si-microstrips (each of $121 \mu \mathrm{m}$ size) is read by dedicated electronic devices (TA1 chips). However, the capacitive coupling between contiguous strips allows not to loose any information. In the Monte Carlo code the capacitive coupling is simulated calculating the total energy release in every readout strip by the weighted sum as obtained in Ref. [7]. For the $k$ th readout strip, the total energy release taking into account neighbour strips is $E_{s}(k)=E_{i}+0.38\left(E_{i-1}+\right.$ $\left.E_{i+1}\right)+0.115\left(E_{i-2}+E_{i+2}\right)+0.095\left(E_{i-3}+E_{i+3}\right)$ $+0.045\left(E_{i-4}+E_{i+4}\right)+0.035\left(E_{i-5}+E_{i+5}\right)$, where $E_{i}$ is the energy release in the $i$ th strip that can be either readout or floating, and $k=1+(i-1) * 2$. This scheme for the capacitive coupling simulation is in agreement with test-beam results obtained from our group for a variety of incidence angles [6].

Instrumental noise was simulated as a Gaussian distribution with $\sigma=5 \mathrm{keV}$. We found that for a detection threshold of $\frac{1}{4} \mathrm{MIP} \simeq 27 \mathrm{keV}$ it has no macroscopic effects on the trigger rates. All the simulation results presented in this document have been obtained using the geometry model described above, taking into account the capacitive coupling and the floating strip readout, but without considering the noise that has negligible effects on the results.

\section{Level-1 trigger}

The Level-1 trigger for the Agile GRID is a hardware-implemented fast logic required to be very rapid $(\sim 2 \mu \mathrm{s})$, conceptually simple, easily implementable by a dedicated hardware, and using parameters reconfigurable by Telecommands. With Monte Carlo simulations we tested different trigger configurations, using the event classes (cosmic gamma-ray photons, background particles and Earth albedo-photons) described in Section 2. Here we present the main results.

Our best strategy for the Level-1 logic is based on the combined use of signals from AC panels and of the ratio $R$, defined as the ratio between the total number of fired TA1 and the total number of fired $X$ and $Y$ views: $R=$ (total no. of fired TA1)/ (total no. of fired $X$ and $Y$ views). In what follows, we call " $R$-trigger" the logic (implemented by a dedicated electronic component) regulating the use of both AC panels and $R$ threshold values.

\subsection{Level-1 trigger configurations}

Here is a short description of the different Level1 trigger configurations that we studied: 
- PLA: events which give hits in at least 3 out of 4 consecutive planes ( $X$ OR $Y$ view),

- TOP: PLA events which pass the TOP AC veto,

- LAT: TOP events with signals in 0 or 1 lateral AC panels, and TOP events which give signals in 2 consecutive AC panels or in 2 AC panels on the same side,

- LSI: LAT events with signals in 0 lateral AC panels, and LAT events with signals in 1 or 2 AC panels but with NO signal in the last silicon plane,

- R11G: LAT events with signals in 0 lateral AC panels, and LAT events with signals in 1 or 2 AC panels and $R>1.1$.

PLA, TOP and LAT are consecutive steps, while LSI and R11G are the two final alternative trigger configurations. For comparison, in Tables 2-4 we reported also the number of events characterized by primary particles or photons reaching the tracker volume (TRA), a box of $38.06 \times 38.06 \times$ $21.078 \mathrm{~cm}^{3}$ which includes the tracker planes from the top sheet of the first tungsten layer to the bottom sheet of the last silicon-y plane.

In the case of gamma-ray photons it is important to observe that:

(1) gamma-photons giving a signal in the tracker may convert in the tracker volume, or in the
Super-AGILE volume (mask or collimators). They can also convert elsewhere (mechanical structure, Mini-Calorimeter or bus MITA), or pass through the instrument without converting. We define as "good photons", those for which there is high probability to reconstruct the incident direction. By definition they are "tracker-converted photons";

(2) because of the presence of the Super-AGILE structure, some gamma-photons potentially able to enter into the Si-Tracker fiducial volume, because of their incidence angles and energies, can convert in the Super-AGILE structure. Their secondaries (electron and positron) may never arrive into the tracker volume. On the other hand, it can happen that some photons, that geometrically would not enter into the tracker volume, convert in the Super-AGILE structure, and their secondaries give a signal in the tracker then inducing a GRID background.

It is then useful to indicate with the suffix "TC" the photons converted in the tracker volume, and with the suffix "SC" the photons converted in the Super-AGILE structure. In the case of gamma-ray photons we also distinguish: (a) photons that theoretically, from a geometrical point of view, might enter into the tracker volume (TRA-TH),

Table 2

Level-1 trigger selection for background charged particles

\begin{tabular}{|c|c|c|c|c|c|}
\hline & $\begin{array}{l}\operatorname{ELE}\left(s^{-1}\right) \\
\text { (percent) }\end{array}$ & $\begin{array}{l}\operatorname{POS}\left(\mathrm{s}^{-1}\right) \\
\text { (percent) }\end{array}$ & $\begin{array}{l}\mathrm{HE} P R O T\left(\mathrm{~s}^{-1}\right) \\
\text { (percent) }\end{array}$ & $\begin{array}{l}\mathrm{LE} \mathrm{PROT}\left(\mathrm{s}^{-1}\right) \\
\text { (percent) }\end{array}$ & $\begin{array}{l}\text { TOTAL }\left(\mathrm{s}^{-1}\right) \\
\text { (percent) }\end{array}$ \\
\hline TRA & 787 & 688 & 123 & 147 & 1745 \\
\hline PLA & 207 & 241 & 82 & 87 & 617 \\
\hline ( $\%$ of TRA) & 26 & 35 & 67 & 59 & 35 \\
\hline TOP & 143 & 151 & 44 & 52 & 390 \\
\hline ( $\%$ of TRA) & 18 & 22 & 36 & 35 & 22 \\
\hline LAT & 120 & 98 & 19 & 28 & 265 \\
\hline ( $\%$ of TRA) & 15 & 14 & 15 & 19 & 15 \\
\hline LSI & 82 & 60 & 2 & 9 & 153 \\
\hline ( $\%$ of TRA) & 10 & 9 & 1 & 6 & 9 \\
\hline $\mathrm{R} 11 \mathrm{G}$ & 55 & 54 & 4 & 6 & 119 \\
\hline ( $\%$ of TRA) & 7 & 8 & 3 & 4 & 7 \\
\hline
\end{tabular}


(b) primary photons actually entered in the tracker volume (PTRA), (c) primary photons converted in the tracker volume (PTCON).

\subsection{Results and discussion}

Tables $2-4$ and Fig. 5 summarize our results in terms of alternative or progressive event selections. The TOP AC veto is useful to reject downgoing

Table 3

Level-1 trigger selection for background albedo-photons

\begin{tabular}{lcc}
\hline & ALB-1 PHOT $\left(\mathrm{s}^{-1}\right)$ & ALB-2 PHOT $\left(\mathrm{s}^{-1}\right)$ \\
\hline TRA & 748 & 1292 \\
PLA & 30 & 48 \\
TOP & 25 & 45 \\
LAT & 24 & 44 \\
LSI & 22 & 41 \\
R11G & 22 & 39 \\
\hline
\end{tabular}

charged particles; the LAT AC veto discriminates events giving a signal either entering than exiting the detector (charged particles) from events which give signals only when they escape from the Tracker (photons into charged pairs). The $R$ trigger is a method optimized to reject noninteracting charged particles, based on a simplified idealization: when a non-interacting particle enters in the Tracker, only one track is expected; if the detector is crossed by a photon creating an electron-positron pair, two tracks are expected. Simulation results show that the $R$-trigger works very well with protons, and gives acceptable results also for electrons and positrons. In general, the method succeeds in discriminating photons from charged background particles very efficiently, sometimes better than the AC system. Clearly, the use of the $R$-trigger does not reject protons generating many secondaries or $\mathrm{e}^{+} / \mathrm{e}^{-}$producing

Table 4

Level-1 trigger selection for cosmic gamma-rays ${ }^{\mathrm{a}}$

\begin{tabular}{|c|c|c|c|c|}
\hline & $\begin{array}{l}\text { PHOT HE 0-10 } \\
\text { (event no.) }\end{array}$ & $\begin{array}{l}\text { PHOT HE 50-60 } \\
\text { (event no.) }\end{array}$ & $\begin{array}{l}\text { PHOT LE 0-10 } \\
\text { (event no.) }\end{array}$ & $\begin{array}{l}\text { PHOT LE 50-60 } \\
\text { (event no.) }\end{array}$ \\
\hline TRA_TH & 3144 & 3352 & 3144 & 3352 \\
\hline TRA & 3511 & 3744 & 3433 & 3646 \\
\hline PTRA & 2975 & 3054 & 3009 & 3175 \\
\hline PTCON & $1488(47 \%)$ & $1292(39 \%)$ & $982(31 \%)$ & $892(27 \%)$ \\
\hline PLA & 1683 & 1415 & 1171 & 908 \\
\hline PLA_TC & $1434(46 \%)$ & $1102(33 \%)$ & $904(29 \%)$ & $694(21 \%)$ \\
\hline PLA_SC & 168 & 128 & 95 & 44 \\
\hline TOP & 1608 & 1355 & 1134 & 882 \\
\hline TOP_TC & $1407(45 \%)$ & $1080(32 \%)$ & $895(28 \%)$ & $685(20 \%)$ \\
\hline TOP_SC & 156 & 121 & 91 & 40 \\
\hline LAT & 1578 & 1213 & 1129 & 872 \\
\hline LAT_TC & $1383(44 \%)$ & $1006(30 \%)$ & $891(28 \%)$ & $681(20 \%)$ \\
\hline LAT_SC & 150 & 102 & 90 & 40 \\
\hline LSI & 1243 & 829 & 1071 & 833 \\
\hline LSI_TC & $1117(36 \%)$ & $690(20 \%)$ & $844(27 \%)$ & $652(19 \%)$ \\
\hline LSI_SC & 94 & 77 & 89 & 40 \\
\hline $\mathrm{R} 11 \mathrm{G}$ & 1469 & 1029 & 998 & 766 \\
\hline $\mathrm{R} 11 \mathrm{G}_{-} \mathrm{TC}$ & $1258(40 \%)$ & $858(26 \%)$ & $803(26 \%)$ & $606(18 \%)$ \\
\hline R11G_SC & 136 & 84 & 72 & 36 \\
\hline
\end{tabular}

\footnotetext{
${ }^{\mathrm{a}}$ In parenthesis we reported the percentage of selected events respect to the total number of photons that theoretically could enter into the tracker volume ( $\%$ of TRA_TH).
} 

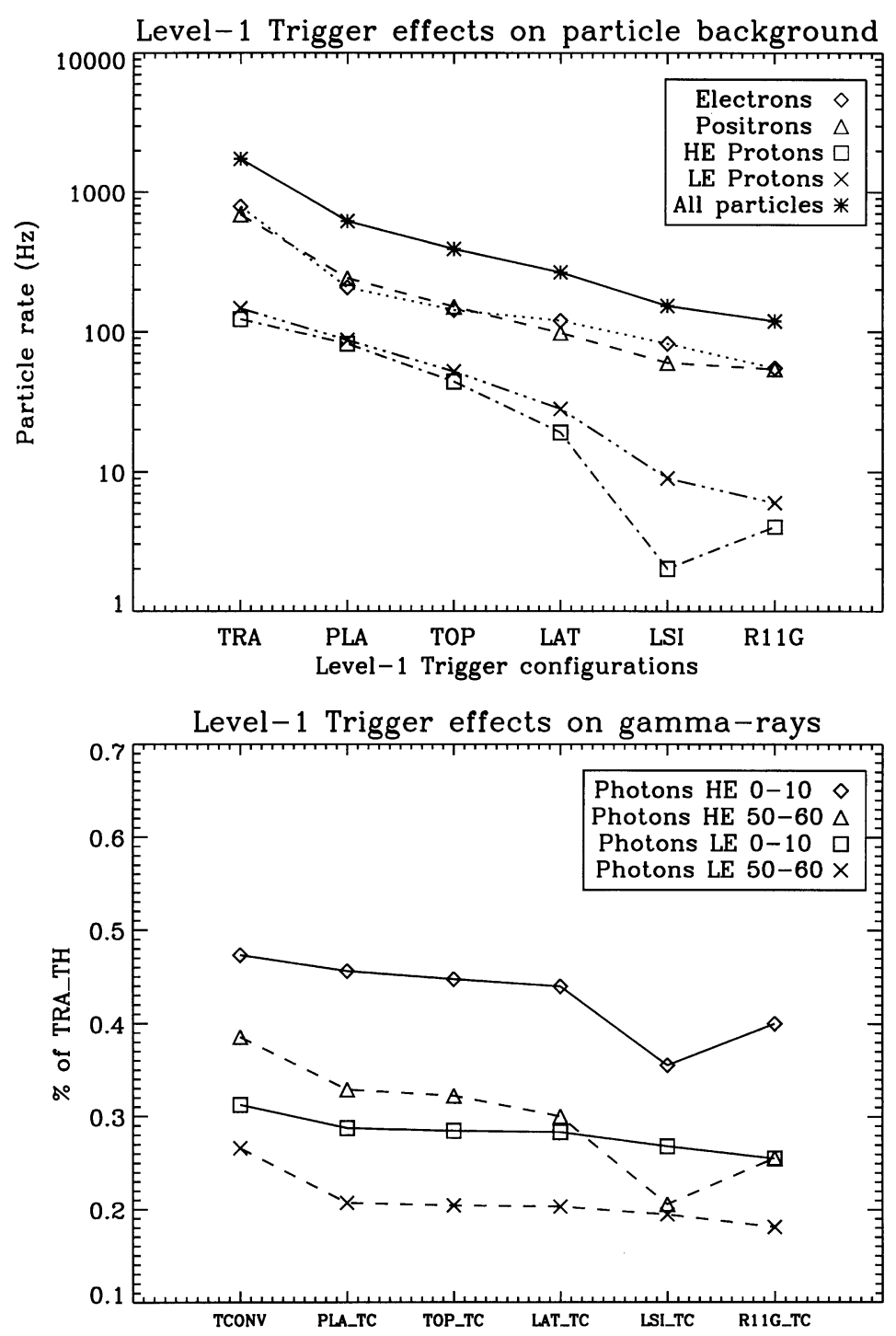

Fig. 5. Particle background rates for different Level-1 trigger configurations and Level-1 trigger efficiency for different photon classes. Event selection based on TRA, PLA, TOP and LAT are sequentially applied to all events. Further selections based on LSI or R11G are instead mutually exclusive. The suffix "TC" denotes "Tracker-converted".

secondaries and electromagnetic showers. A trigger configuration based only on the AC system does not reject low energy protons stopping inside the tracker. The two methods are based on different "philosophies" and they both give good results in different situations.

From our simulations it appears that the best choice is a combination of the two strategies and that the most efficient Level-1 trigger configura- tion is $\mathrm{R} 11 \mathrm{G}$, rejecting $\sim 93 \%$ of background particles without affecting significantly the gamma-ray detection rate.

\section{Level-1.5 data processing}

The additional "Level-1.5" data processing uses the tracker discretization in terms of TA1 chips 
$\left(3.1 \times 3.1 \mathrm{~cm}^{2}\right.$ square elements), together with the complete $\mathrm{AC}$ and $\mathrm{MC}$ information. We investigated the efficiency of alternative Level-1.5 Trigger options in rejecting background charged particles and here we present the main results.

\subsection{Level 1.5 trigger configurations}

We considered the following options:

- DIS: The DIS option is a simplified track reconstruction and is based on computing the distance $D$ of the fired TA1s from the fired AC lateral panel. The basic idea is that a charged particle is expected to have increasing $D$ values as a function of increasing plane, while a gamma-ray photon is expected to have a decreasing $D$ function (in the restricting hypothesis that they both come downward and in the absence of strong "hard" scattering of photon-created pairs). The parameter DIS is defined as: DIS $=D_{\mathrm{fp}}-D_{\mathrm{lp}}$ where $D_{\mathrm{fp}}$ is the distance of the closest fired TA1 to the fired AC lateral panel in the first plane, while $D_{\mathrm{lp}}$ is the distance of the closest fired TA1 to the fired AC lateral panel in the last plane. We require DIS $\geqslant 0$ for good events. This option is applied only if there are fired AC lateral panels.

- RUD10 (or RUD12): The fired tracker views are divided in two groups (UP and DOWN) and the ratio $R$ is computed separately for the two groups ( $R^{\text {up }}$ and $R^{\text {down }}$ ). The ratio RUD = $R^{\text {up }} / R^{\text {down }}$ can help in discriminating good gamma-photons, that come downward, from secondary photons generated by charged particles, that come from the bottom toward the top of the detector. With the option RUD10 are rejected all the events with RUD $>1.0$, while with the option RUD12 are rejected all the events with RUD $>1.2$.

- VFORM: This option, as RUD10 and RUD12, can help in discriminating primary photons going downward from secondary photons coming upward. It computes the separation $S$ between fired TA1 on the same view, divides the fired planes in two groups (UP and DOWN) and compares the mean separation of the UP planes with the mean separation of the DOWN planes. The parameter $V$ is defined as $V=$ $S_{\text {down }}-S_{\text {up }}$ and events with $V<0$ are rejected.

- CGAP: This option rejects events with a gap of more than 2 planes (4 views) between the MC and the last fired tracker view. It is applied only if there is a signal from the $\mathrm{MC}$ and it is supposed to reject events due to $\mathrm{e}^{+}$or $\mathrm{e}^{-}$which stop in the MC and generate a secondary photon which enters the tracker upward and creates a couple only after passing 4 or more views.

- TGAP: This option rejects events with a gap of more than 2 planes (4 views) in the tracker. The parameter T_GAP is defined, for a single event, as the maximum number of no-fired views between two non-consecutive fired views.

\subsection{Results and discussion on the Level-1.5 trigger processing}

Tables 5 and 6 and Fig. 6 show the simulation results. The simple DIS algorithm is the most efficient in rejecting background particles without

Table 5

Summary of Level-1.5 processing of background events

\begin{tabular}{llllllll}
\hline Type & R11G $\left(\mathrm{s}^{-1}\right)$ & DIS $\left(\mathrm{s}^{-1}\right)$ & RUD12 $\left(\mathrm{s}^{-1}\right)$ & RUD10 $\left(\mathrm{s}^{-1}\right)$ & VFORM $\left(\mathrm{s}^{-1}\right)$ & CGAP $\left(\mathrm{s}^{-1}\right)$ & TGAP $\left(\mathrm{s}^{-1}\right)$ \\
\hline ELE & 55 & 35 & 41 & 36 & 45 & 52 & 46 \\
POS & 54 & 30 & 43 & 36 & 42 & 50 & 43 \\
LE PR. & 6.2 & 3.4 & 4.9 & 4.2 & 3.8 & 3.8 & 5.8 \\
HE PR. & 4.1 & 1.5 & 2.8 & 79 & 96 & 112 & 3.8 \\
Total & 119 & 70 & 92 & 18 & 19 & 35 & 18 \\
ALB-1 & 22 & 20 & 17 & 33 & 35 & 36 & 33 \\
ALB-2 & 39 & 36 & 30 & & & & \\
\hline
\end{tabular}


Table 6

Summary of Level-1.5 processing of cosmic gamma-ray events ${ }^{\mathrm{a}}$

\begin{tabular}{llllllll}
\hline $\begin{array}{l}\text { Photon } \\
\text { class }\end{array}$ & $\begin{array}{l}\text { R11G } \\
(\mathrm{TC})(\%)\end{array}$ & $\begin{array}{l}\text { DIS } \\
(\mathrm{TC})(\%)\end{array}$ & $\begin{array}{l}\text { RUD12 } \\
(\mathrm{TC})(\%)\end{array}$ & $\begin{array}{l}\text { RUD10 } \\
(\mathrm{TC})(\%)\end{array}$ & $\begin{array}{l}\text { VFORM } \\
(\mathrm{TC})(\%)\end{array}$ & $\begin{array}{l}\text { CGAP } \\
(\mathrm{TC})(\%)\end{array}$ & $\begin{array}{l}\text { TGAP } \\
(\mathrm{TC})(\%)\end{array}$ \\
\hline HE 0-10 & 40 & 39 & 39 & 36 & 35 & 39 & 38 \\
HE 50-60 & 26 & 25 & 24 & 21 & 19 & 26 & 23 \\
LE 0-10 & 26 & 26 & 23 & 20 & 25 & 22 & 16 \\
LE 50-60 & 18 & 17 & 14 & 12 & 15 & 15 \\
\hline
\end{tabular}

${ }^{a}$ Simulation results concerning only Tracker-Converted (TC) photons. Values in the table represent the percentage of the number of simulated events that pass the different Level-1.5 trigger options respect to the total number of photons that theoretically could enter into the Tracker volume ( $\%$ of TRA_TH).
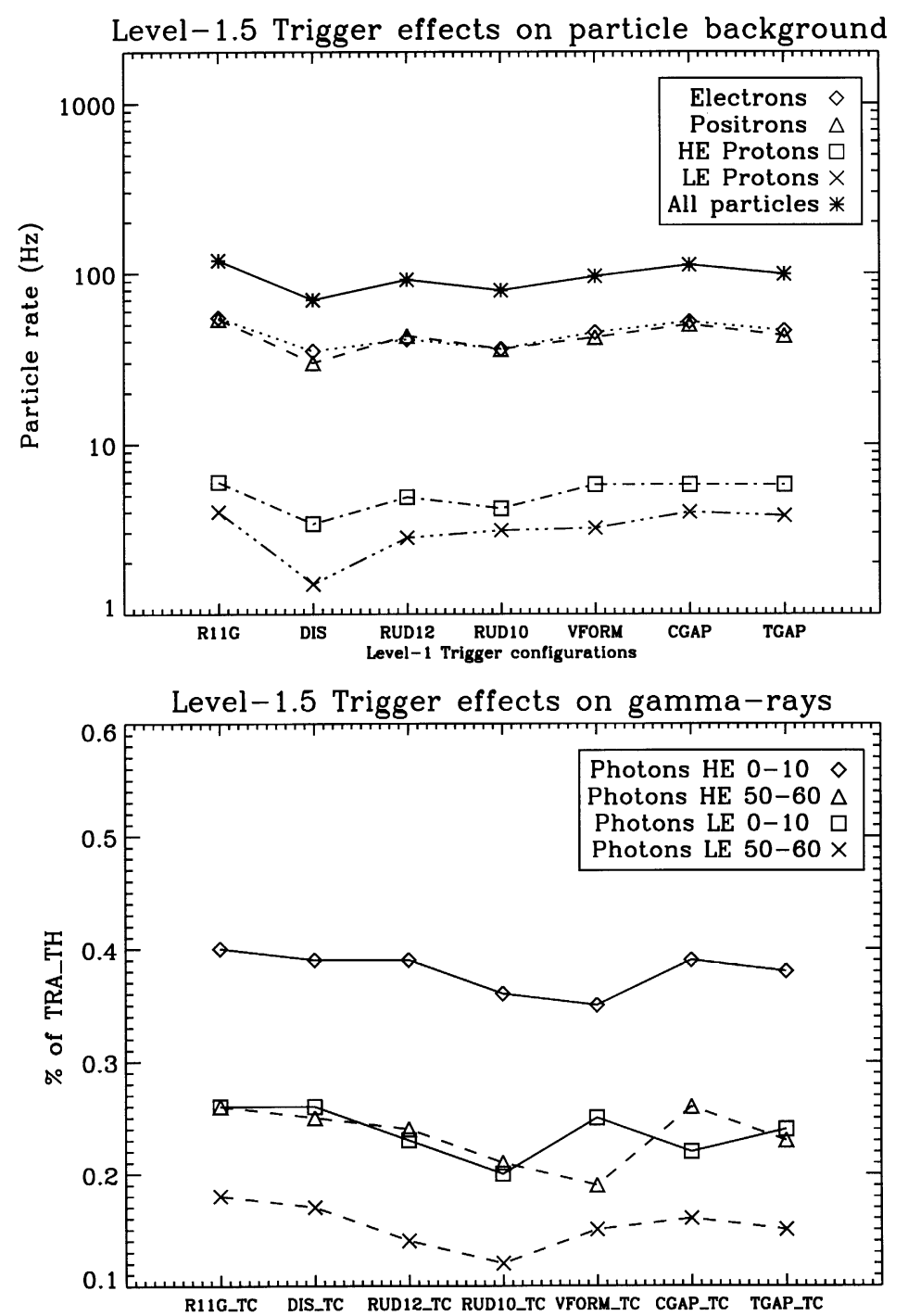

Fig. 6. These plots show the efficiency of the Level-1.5 trigger alternative options in rejecting particle background and in detecting gamma-photons (DISRUD means (DIS+RUD_10) and DISCG means (DIS + C_GAP)). 
losing too many good photons. This algorithm is applied only if there is at least one fired AC lateral panel; most of background charged particles verify this condition, while good photons often do not hit any lateral AC panel.

Options RUD10, RUD12 and VFORM are not very efficient because the distributions of the RUD and $V$ parameters are not significantly different for background particles and for good photons. The C_GAP option is not efficient because the majority of background electrons, positrons and albedo protons do not give a signal in the MiniCalorimeter. There are no significant differences between the background particle T_GAP distributions and the photon T_GAP distribution, probably because this parameter is influenced by secondary particles.

\section{Discussion and conclusions}

By using a complete and detailed model of the AGILE instrument, we simulated with GEANT the performance of the on-board Level-1 trigger logic. We obtain satisfactory results from our optimization study of the Level-1 and 1.5 trigger logic for the cosmic gamma-ray signal (with photon energies between $\sim 30 \mathrm{MeV}$ and $50 \mathrm{GeV}$ ) and for the rejection of the particle and albedophoton backgrounds in a $550 \mathrm{~km}$ equatorial orbit. The optimal Level-1 (and therefore necessarily simple) event selection algorithms are the R11G and DIS procedures that can be easily hardwareimplemented and are very fast $(\leqslant 20 \mu$ s). Table 7

Table 7

Average GRID Background Event Rates after Level-1+1.5 Processing

\begin{tabular}{lll}
\hline Background component & $\begin{array}{l}\text { Unocculted } \\
\text { GRID FOV } \\
\left(\mathrm{s}^{-1}\right)\end{array}$ & $\begin{array}{l}\text { Half-occulted } \\
\text { GRID FOV } \\
\left(\mathrm{s}^{-1}\right)\end{array}$ \\
\hline Charged particles & 70 & 70 \\
Earth albedo-photons & 20 & 40 \\
Total & 90 & 110 \\
\hline
\end{tabular}

summarizes the main results of our paper: the total background rates expected for two different Earth occultation geometries. At the Level- $1+1.5$ the background component completely dominates the event rate over the cosmic gamma-rays by a factor between 10 and 100, and the total background event rate is near $100 \mathrm{~s}^{-1}$, a value which can be sustained by the on-board AGILE Data Handling [2]. Therefore the Level-1 logic presented here is adequate for the AGILE GRID.

We also note that the albedo-photons contribute a significant fraction (between $\sim 30 \%$ and more than $\sim 50 \%$ depending on the Earth's position with respect to the GRID) of the total background rate. Since these photons are dominated by 10 $100 \mathrm{MeV}$ gamma-rays, Level-1 techniques are not adequate in substantially reducing their event rate, and a special Level-2 data processing is required, which will be the subject of a forthcoming paper.

Our results are of general interest for space detectors similar to the AGILE GRID. Two conclusions are of particular interest: (1) the fact that low-energy leptons (magnetospheric trapped electrons and positrons) dominate the particle background requires special attention and a proper event selection logic; (2) Earth albedophotons constitute a substantial fraction of the residual events passing the Level-1 trigger stage.

\section{Acknowledgements}

Results presented in this paper are based on joint work with members of the AGILE Team. In particular, we warmly thank G. Barbiellini, P. Picozza and A. Morselli for special support, M. Prest, E. Vallazza and G. Fedel for exchange of information on the Silicon Tracker performance and experimental data, and the AGILE Simulation and Theory Group for many discussions. We also thank A. Pellizzoni and P. Lipari for collaboration on several background source function algorithms used in our simulations.

The current work was carried out at the University of Rome "Tor Vergata", University of Ferrara and CNR and INFN laboratories, under the auspices and partial support of the Agenzia Spaziale Italiana. 


\section{References}

[1] M. Tavani, et al., (2002) in preparation.

[2] M. Tavani, et al., Invited paper presented at the Gamma 2001 Symposium, Baltimore, 4-6 April 2001, American Institute of Physics Conference Proceedings, 587 (2001) 729.

[3] M. Tavani, et al., Science with AGILE, AGILE Internal Note, A-P-019, 2001, (http://www.ifctr.mi.cnr.it/Agile).

[4] V. Cocco, F. Longo, M. Tavani, Nucl. Instr. and Meth. A 486 (2002) 623.

[5] R. Brun, et al., GEANT Detector Description and Simulation Tool, 1993, CERN, Geneva.

[6] G. Barbiellini, et al., INFN/TC01/006, Nucl. Instr. and Meth. A (2002) submitted for publication.

[7] G. Fedel, Dissertation for the Laurea in Physics, University of Trieste, 2000.

[8] M. Prest, et al., (2002) in preparation.

[9] C.E. Fichtel, et al., Astrophys. J. 198 (1975) 163.

[10] D. Thompson, 1999, private communication.

[11] M. Feroci, et al., In-flight performances of the BeppoSAX gamma-ray burst monitor, in: Proceedings of the SPIE Conference, 1997, Vol. 3114, San Diego.
[12] R. Battiston, Search for antimatter in space with the Alpha Magnetic Spectrometer, astro-ph/9907152, Presented at 13th Les Rencontres de Physique de la Vallee d'Aoste: "Results and Perspectives in Particle Physics", 1999.

[13] J. Alcaraz, et al., Phys. Lett. B 472 (2000) 215.

[14] J. Alcaraz, et al., Phys. Lett. B 484 (2000) 10.

[15] S.V. Koldashov, et al., Electron and positron albedo spectra with energy more than $10 \mathrm{MeV}$, Proceedings of the 24th ICRC, 1995, Vol. 4, p. 993.

[16] V. Cocco, P. Lipari, F. Longo, Note on the charged particle albedo fluxes, AGILE Technical Note, AGILESIM-TN-002, 2000.

[17] D.J. Thompson, G.A. Simpson, M.E. Ozel, J. Geophys. Res. 86 (1981) 1265.

[18] E. Costa, E. Massaro, M. Salvati, A. Apolloni, Astrophys. Space Sci. 100 (1984) 165.

[19] J.C. Ling, Ph.D. Thesis, 1974, UCSD.

[20] J.L Matteson, J.C. Ling, L.E. Peterson, Proceedings of the Ninth ESLAB Symposium, 1974, p. 137.

[21] V. Schoenfelder, U. Graser, J. Daugherty, Astrophys. J. 217 (1977) 306.

[22] V. Schoenfelder, F. Grami, F.P. Penningsfeld, Astrophys. J. 240 (1980) 350. 